\title{
Investigations on the efficacy of routinely used phenotypic methods compared to genotypic approaches for the identification of staphylococcal species isolated from companion animals in Irish veterinary hospitals
}

\author{
Lisa Geraghty ${ }^{1,2^{*}}$, Mary Booth ${ }^{1,2}$, Neil Rowan ${ }^{1}$ and Andrew Fogarty ${ }^{1,2}$
}

\begin{abstract}
Background: Identification of Staphylococci to species level in veterinary microbiology is important to inform therapeutic intervention and management. We report on the efficacy of three routinely used commercial phenotypic methods for staphylococcal species identification, namely API Staph 32 (bioMérieux), RaplD (Remel) and Staph-Zym (Rosco Diagnostica) compared to genotyping as a reference method to identify 52 staphylococcal clinical isolates (23 coagulase positive; 29 coagulase negative) from companion animals in Irish veterinary hospitals.

Results: Genotyping of a 412 bp fragment of the staphylococcal tuf gene and coagulase testing were carried out on all 52 veterinary samples along with 7 reference strains. In addition, genotyping of the staphylococcal rpoB gene, as well as PCR-RFLP of the pta gene, were performed to definitively identify members of the Staphylococcus intermedius group (SIG). The API Staph 32 correctly identified all S. aureus isolates (11/11), 83\% (10/12) of the SIG species, and 66\% (19/29) of the coagulase negative species. RapID and Staph-Zym correctly identified 61\% $(14 / 23)$ and $0 \%(0 / 23)$ respectively of the coagulase-positives, and 10\% (3/29) and 3\% (1/29) respectively of the coagulase-negative species.

Conclusions: Commercially available phenotypic species identification tests are inadequate for the correct identification of both coagulase negative and coagulase positive staphylococcal species from companion animals. Genotyping using the tuf gene sequence is superior to phenotyping for identification of staphylococcal species of animal origin. However, use of PCR-RFLP of pta gene or rpoB sequencing is recommended as a confirmatory method for discriminating between SIG isolates.
\end{abstract}

Keywords: Companion animals, Staphylococci species identification, Genotyping, tuf, rpoB

\section{Background}

Staphylococcal species are considered to be opportunistic pathogens, colonising the skin and mucous membranes of humans and animals. In animals, both coagulase positive and coagulase negative Staphylococci have been associated with infection, with a variety of sources identified [1,2]. International animal surveillance programmes on emerging

\footnotetext{
* Correspondence: Igeraghty@ait.ie

'Biosciences Research Institute, Athlone Institute of Technology, Dublin Rd., Athlone, Co. Westmeath, Ireland

${ }^{2}$ Department of Life and Physical Science, School of Science, Athlone Institute of Technology, Dublin Rd., Athlone, Ireland
}

trends in antibiotic resistance typically focus on food animals. Consequently, there is a dearth of similar information for pets or companion animals that frequently are administered with antibiotics, particularly in veterinary hospitals. Recent studies have reported a link between the isolation of multi-drug resistant bacteria from pet owners with companion animal carriage [1]. Antibiotic resistance to betalactams, including methicillin resistance, has been found in both coagulase negative and coagulase positive Staphylococci carried by healthy and infected cats, dogs and horses [3-5] reported that various coagulase negative species of 
Staphylococci from bovine milk differed significantly from each other in antimicrobial resistance profiles (both genotypic and phenotypic) with implications for treatment and management decisions. Accurate speciation of Staphylococci is vital to establish critical links between bacterial species of clinical origin and emerging trends in antibiotic resistance.

Historically, identification of bacterial specimens has been based on conventional microbiological procedures, which include growth on various media, cell morphology, staining reactions and biochemical profiles [6]. Currently in Ireland commercial systems such as API Staph 32 (bioMérieux), Rap-ID (Remel) and Staph-Zym (Rosco Diagnostica) are regularly used to identify species of Staphylococci. There is an absence of published information on the efficacy of using these commercial phenotypic methods for routine Staphylococcal identification particularly from companion animals. Recent reports suggest that phenotypic methods have inherent weaknesses due to the variability in expression of phenotypic characteristics by isolates belonging to the same species and their reliance on subjective interpretation of test results that can also introduce variability [7-11]. Blaiotta and co-workers $[8,12]$ reported a large variation in phenotypic properties of Staphylococci isolated from fermented sausages using laboratory-prepared basal media supplemented separately with fermentable sugars. This group also reported that $25 \%$ of these Staphylococci were not identifiable using phenotypic methods. Most phenotypic identification systems have been developed for human healthcare and validated using clinical isolates obtained from human infections $[9,11]$. When employed on isolates of animal origin, the identification system may be less reliable due to the lack of animal isolates in the reference databases $[13,14]$.

Genotypic methods are reported to have higher discriminatory power, reproducibility and typeability compared to phenotypic methods $[10,11,15,16]$. Several approaches are available for genotyping bacterial isolates including AFLP (amplified fragment length polymorphism), ribotyping, PCR-RFLP and DNA sequencing. DNA sequence based species identification of Staphylococci is currently the most accurate method with the largest reference database, and is considered to be the gold standard method $[10,11]$. Traditionally, the most common target for DNA sequencing in bacteria is 16S-rRNA [11,17]. However, this gene is highly conserved among Staphylococci, and often does not provide sufficient discriminatory power to differentiate closely related staphylococcal species [11]. Alternatives to the $16 \mathrm{~S}$ rRNA gene which have been successfully applied to staphylococcal genotyping include gap [17], cpn 60 [18], tuf [9], rpoB [19], nuc [20] and sodA [21]. Heikens et al., [9] first proposed partial amplification and sequencing of the tuf gene as a reliable and reproducible method for the identification of species of Staphylococci. Subsequent studies have confirmed tuf gene sequencing as an accurate method for speciating coagulase negative Staphylococci, [22,23]. Blaiotta et al. [24] revealed diversity among coagulase positive Staphylococcus species strains based on partial kat (catalase) gene sequences and reported a PCR-RFLP assay for identification of coagulase-positive species (S. aureus, S. delphini, S. hyicus, S. intermedius, S. pseudomedius, S. schleiferi subsp. coagulans). Similarly, Sasaki et al. [25] used a multiplexPCR method targeting the staphylococcal thermonuclease gene $(n u c)$ to successfully differentiate between the same species.

Based upon best-published literature, there is a dearth of critical information on the efficacy of using phenotypic "quick tests" to identify staphylococcal species associated with companion animals. Therefore, this study aimed to compare the efficacy of three routinely-used phenotypic staphylococcal identification test kits with genotypic methods in order to identify the most accurate method of speciating clinical isolates of Staphylococci from a variety of companion animals (dogs, cats, horses) in primary care veterinary hospitals throughout Ireland.

\section{Methods}

\section{Bacterial strains}

Fifty two staphylococcal clinical isolates of veterinary origin and 7 reference control strains were analysed in this study. Clinical specimens were isolated from infection site swabs taken at primary care veterinary clinics (Table 1). Animal species included feline $(n=21)$, canine $(\mathrm{n}=18)$, equine $(\mathrm{n}=11)$ and bovine $(\mathrm{n}=2)$. Infection types included flesh wounds and superficial abscesses $(\mathrm{n}=38)$, deep seated infections $(n=9)$, post-operative infections $(\mathrm{n}=4)$, and ocular infections $(\mathrm{n}=3)$. Swabs were streaked onto Columbia blood agar and Staphylococci were identified by colony morphology, haemolysis patterns, Gram's stain characteristics, catalase activity, growth on Baird Parker agar, coagulase activity and Voges-Proskauer (VP) testing. Two different coagulase tests were performed: a tube test for free coagulase and a slide test for bound coagulase of clumping factor. Seven control strains and fiftytwo clinical isolates were tube coagulase tested according to Murray et al. [6] as follows: a mixture of a $0.1 \mathrm{ml}$ nutrient broth incubated with overnight culture was mixed with $0.5 \mathrm{ml}$ of reconstituted rabbit plasma containing EDTA (Rabbit Coagulase Plasma, Cruinn Diagnostics) in a sterile glass tube and incubated at $37^{\circ} \mathrm{C}$ in a water bath for $4 \mathrm{hrs}$. The tubes were observed for clot formation by gently tilting at a $90^{\circ}$ angle from the vertical. The tubes were then re-incubated and re-read at 24 hrs. Any degree of clotting was read as a positive result. The slide coagulase test was performed using a Staphylase test kit (Oxoid) according to manufacturer's instructions. A positive result 
Table 1 Source of Staphylococci isolated from companion animals in veterinary hospitals

\begin{tabular}{|c|c|c|c|c|}
\hline $\begin{array}{l}\text { Isolate } \\
\text { no. }\end{array}$ & $\begin{array}{l}\text { Staphylococcal } \\
\text { species }\end{array}$ & $\begin{array}{l}\text { Companion } \\
\text { animal }\end{array}$ & Infection type & $\begin{array}{c}\text { Location of } \\
\text { veterinary } \\
\text { hospital } \\
\text { (Region of Ireland) }\end{array}$ \\
\hline 1 & S. aureus & equine & Joint infection & north \\
\hline 2 & S. aureus & feline & Flesh wound & north \\
\hline 3 & S. aureus & feline & Face abscess & west \\
\hline 4 & S. aureus & bovine & Post caesarian & west \\
\hline 5 & S. aureus & feline & Interdigital infection & west \\
\hline 6 & S. aureus & feline & Interdigital infection & west \\
\hline 7 & S. aureus & equine & Abdominal trauma & south \\
\hline 8 & S. aureus & canine & Post ortho op & east \\
\hline 9 & S. aureus & feline & Cat fight abscess & west \\
\hline 10 & S. aureus & feline & Flesh wound & west \\
\hline 11 & S. aureus & equine & Flesh wound & west \\
\hline 12 & S. pseudintermedius & canine & Post ortho op & west \\
\hline 13 & S. pseudintermedius & canine & Granuloma type & north \\
\hline 14 & S. pseudintermedius & canine & Pyoderma & north \\
\hline 15 & S. pseudintermedius & canine & Flesh wound & north \\
\hline 16 & S. pseudintermedius & feline & Deglove wound & west \\
\hline 17 & S. pseudintermedius & canine & Flesh wound & south \\
\hline 18 & S. pseudintermedius & canine & Flesh trauma & west \\
\hline 19 & S. pseudintermedius & equine & Abdominal wound & south \\
\hline 20 & S. pseudintermedius & equine & Joint infection & north \\
\hline 21 & S. pseudintermedius & canine & Ear tip infection & west \\
\hline 22 & S. pseudintermedius & feline & Granuloma type & east \\
\hline 23 & S. pseudintermedius & canine & Flesh wound & south \\
\hline 24 & S. felis & canine & Flesh wound & north \\
\hline 25 & S. felis & feline & Ocular infection & east \\
\hline 26 & S. felis & canine & Post hysterectomy & west \\
\hline 27 & S. equorum & canine & Eyelid infection & south \\
\hline 28 & S equorum & canine & Flesh wound & north \\
\hline 29 & S. equorum & canine & Face pyoderma & north \\
\hline 30 & S. equorum & canine & Ear infection & north \\
\hline 31 & S. equorum & feline & Trauma to face & south \\
\hline 32 & S. equorum & feline & Cat fight abscess & south \\
\hline 33 & S equorum & equine & Uterine infection & east \\
\hline 34 & S. equorum & feline & Flesh wound & south \\
\hline 35 & S. succinus & equine & Uterine infection & west \\
\hline 36 & S. warneri/pasteuri & canine & Eyelid infection & south \\
\hline 37 & S. warneri/pasteuri & bovine & Chronic mastitis & west \\
\hline 38 & S. carnosus/simulans & feline & Abscess & south \\
\hline 49 & S. carnosus & feline & Rhinitis & north \\
\hline 40 & S. carnosus & feline & Pyoderma & north \\
\hline 41 & S. carnosus /simulans & feline & Puncture wound & west \\
\hline 42 & S. carnosus/simulans & equine & Chronic flesh wound & west \\
\hline 43 & S. carnosus/simulans & feline & Abscess & south \\
\hline 44 & S. carnosus & feline & Ear infection & west \\
\hline 45 & S. carnosus/simulans & feline & Puncture wound & west \\
\hline
\end{tabular}


Table 1 Source of Staphylococci isolated from companion animals in veterinary hospitals (Continued)

\begin{tabular}{|c|c|c|c|c|}
\hline 46 & S. carnosus /simulans & equine & Uterine infection & east \\
\hline 47 & S. xylosus & feline & Laceration flesh & east \\
\hline 48 & S. xylosus & canine & Flesh wound & east \\
\hline 49 & S. xylosus & equine & Uterine infection & west \\
\hline 50 & S. xylosus & canine & Deglove RTA & south \\
\hline 51 & S. saprophyticus & feline & Flesh wound & north \\
\hline 52 & S saprophyticus & feline & Infection in paw & south \\
\hline
\end{tabular}

was read when clumping occurred within 10 seconds. Reference strain identity and source are shown in Table 2.

\section{Phenotypic identification testing}

Phenotypic identification to species level was carried out using three test kits which are commercially available to veterinary laboratories in Ireland, i.e. API Staph 32 (bioMérieux), RapID (Remel) and Staph-Zym (Rosco Diagnostica). Prior to testing, isolates were cultured overnight at $37^{\circ} \mathrm{C}$ on Columbia blood agar. Tests were carried out according to manufacturers' instructions and results were interpreted using the appropriate laboratory computer software or reference indices recommended by the manufacturer. The results of the phenotypic tests in this study are also described in terms of sensitivity, specificity and predictive value positive (PVP) of the three test kits and were calculated in comparison with tuf genotyping [5]. Sensitivity was calculated as the proportion of the true positive isolates that are correctly identified with the phenotypic tests. Specificity was calculated as the proportion of the true negatives that are correctly identified with the phenotypic tests. The predictive value positive (PVP) for each test was calculated as the proportion of isolates identified as a specific species based on phenotypic testing that truly represented that particular species.

\section{Genotypic identification testing}

Genomic DNA from overnight liquid cultures in nutrient broth was extracted using a DNeasy kit (QIAGEN) according to manufacturer's instructions. For genotyping, a 412 bp fragment of the tuf gene was amplified for all clinical isolates and reference strains according to Heikens et al. [9]. Amplification of a 750 bp rpoB gene segment was carried out on all SIG isolates (3 reference strains and 12 clinical isolates) according to Drancourt and Raoult [19], with the following modifications in PCR cycling conditions: 2 minutes at $95^{\circ} \mathrm{C}$ for 1 cycle, 30 seconds at $94^{\circ} \mathrm{C}$, 30 seconds at $47^{\circ} \mathrm{C}, 1$ minute at $72^{\circ} \mathrm{C}$ for 35 cycles, and 5 minutes at $72^{\circ} \mathrm{C}$ for 1 cycle.

\section{DNA sequencing}

A total of 59 tuf amplicons (from 7 reference and 52 clinical isolates) and 15 rpoB amplicons (3 reference and 12 clinical isolates) were sequenced by Sequiserve, Germany and Functional Biosciences, USA using amplification primers $[9,19]$. Forward and reverse sequences were analysed using the BLASTn alignment program and the NCBI nucleotide database NCBI [26].

\section{PCR-RFLP to differentiate SIG species}

Twelve clinical isolates were identified by both tuf and rpoB genotyping as members of the Staphylococcus intermedius group (SIG). PCR-RFLP involving amplification of the pta

Table 2 Identification of staphylococcal reference strains

\begin{tabular}{|c|c|c|c|c|c|c|c|}
\hline Species ID & Source* & tuf & rpoB & pta-RFLP & $\begin{array}{c}\text { API Staph } \\
32\end{array}$ & RapID & $\begin{array}{l}\text { Staph- } \\
\text { Zym }\end{array}$ \\
\hline S. aureus IMD247 & $\begin{array}{c}\text { Athlone Institute of } \\
\text { Technology }\end{array}$ & S. aureus & - & - & S. aureus & S. aureus & S. vitulans \\
\hline S. hycius 11249 & University College Dublin & S. hycius & - & - & S. hycius & S. aureus & S. hycius \\
\hline S. aureus 25923 & ATCC & S. aureus & - & - & S. aureus & S. aureus & No result \\
\hline S. aureus 43300 & ATCC & S. aureus & - & - & S. aureus & S. aureus & S. vitulans \\
\hline $\begin{array}{l}\text { S. intermedius CCUG } \\
6520\end{array}$ & University of Copenhagen & S. intermedius & S. intermedius & S. intermedius & $\begin{array}{c}\text { S. } \\
\text { intermedius }\end{array}$ & $\begin{array}{c}\text { S. } \\
\text { intermedius }\end{array}$ & No result \\
\hline S. delphini M4 & University of Copenhagen & S. delphini & S. delphini & S. delphini & $\begin{array}{c}\text { S. } \\
\text { intermedius }\end{array}$ & No result & S. vitulans \\
\hline $\begin{array}{c}\text { S. pseudinter-medius } \\
\text { Y19 }\end{array}$ & University of Copenhagen & $\begin{array}{l}\text { S. pseudinter - } \\
\text { medius }\end{array}$ & $\begin{array}{l}\text { S. pseudinter- } \\
\text { medius }\end{array}$ & $\begin{array}{l}\text { S. pseudinter- } \\
\text { medius }\end{array}$ & $\begin{array}{c}\text { S. } \\
\text { intermedius }\end{array}$ & $\begin{array}{c}\text { S. } \\
\text { intermedius }\end{array}$ & S. vitulans \\
\hline
\end{tabular}

*American Typed Culture Collection (ATCC). 
gene followed by digestion with Alu1 was used in this study to distinguish between the three known species in the SIG group according to Bannhoer et al., [27] and Kadlec et al. [28].

\section{Results \\ Comparative use of phenotypic 'quick tests' and genotypic methods to identify reference strains of staphylococcal species}

Seven reference staphylococcal strains were analysed in this study using each of the speciation methods: tuf genotyping, API Staph 32, RapID and Staph-Zym. The results are displayed in Table 2 . The tuf genotyping correctly identified all reference strains. The API Staph 32 test correctly identified S. aureus, S. hycius, and S. intermedius reference strains. However S. pseudintermedius and S. delphini, were both misidentified indicating that this test does not distinguish SIG species. The RapID test correctly identified all of the $S$. aureus reference strains and $S$. intermedius, but misidentified S. hycius and S. pseudintermedius. RapID gave no result for $S$. delphini. Staph-Zym identified only one (S. hycius) of the 7 control strains, correctly. The three SIG reference strains in the study were also analysed by rpoB genotyping and PCR-RFLP of the pta gene and were correctly identified by both tests.

\section{Speciation of staphylococcal clinical isolates}

Based on using tuf genotyping as the reference identification method for this study, the clinical isolate collection $(n=52)$ was found to comprise of a range of staphylococcal species. The results are shown in Table 3. Forty four percent $(23 / 52)$ were identified as coagulase positive species, of which $47.8 \%(11 / 23)$ were $S$. aureus and $43 \%(10 / 23)$ were $S$. pseudintermedius (Table 3 ). For two of the coagulase positive isolates, tuf did not distinguish between S. pseudintermedius and S. delphini (Isolate No's. 18 and 23). All twelve SIG clinical isolates were confirmed to be $S$. pseudintermedius according to banding patterns using PCR-RFLP of the pta gene and rpoB genotyping. Fifty six percent (29/52) of isolates were identified by tuf genotyping as coagulase negative Staphylococci (CONS) (Table 3). These included, S. equorum $(\mathrm{n}=8)$, S. xylosus $(\mathrm{n}=4)$, S. carnosus/simulans $(\mathrm{n}=6)$, S. carnosus $(\mathrm{n}=3)$, S. felis $(\mathrm{n}=3)$, S. warneri/pasteuri $(\mathrm{n}=2)$, S. saprophyticus $(\mathrm{n}=2)$ and $S$. succinus $(\mathrm{n}=1)$. For six of the CONS isolates, tuf genotyping did not distinguish between two closely related coagulase negative species, identifying them as $S$. carnosus or S. simulans, a finding which correlates with research carried out by Ghebremedhin et al. [29]. For two additional isolates a result of S. pasteuri or S. warneri was obtained. The results of tuf genotyping were consistent with the coagulase test results in all cases with five exceptions, namely $S$. aureus (Isolate No. 1) and S. pseudintermedius (Isolate No's 16, 17, 22 and 23). These strains failed to coagulate plasma in both slide and tube coagulation tests, but were subsequently confirmed as coagulase positive species by PCR-RPLP of the pta gene and rpoB sequencing.

The results of the phenotypic tests in this study are summarised in Table 4, where the sensitivity, specificity and predictive value positive (PVP) of the three test kits are calculated in comparison with tuf species identification. The API Staph 32 test showed greatest sensitivity for S. aureus isolates (100\%). A majority (10/12) of the $S$. pseudintermedius isolates were positively identified as members of the SIG group (83.3\%), however, none were accurately speciated. The remaining $S$. pseudintermedius isolates gave either no result $(n=1)$ or was misidentified $(\mathrm{n}=1)$. Specificity was $100 \%$ for S. aureus, and $95 \%$ for the SIG group. For the remaining 29 isolates, which were CONS, 31\% (9/29) were correctly identified. Isolates identified by tuf genotyping as $S$. carnosus/simulans were correctly identified by API Staph 32 as either S. carnosus or S. simulans in six out of nine cases (66.6\% sensitivity). S. xylosus species were correctly identified by API Staph 32 with a sensitivity of $75 \%$. In this test, specificity for these two species was 95.3 and $83.3 \%$ respectively. Sensitivity was $0 \%$ for S. equorum, S. felis and S. succinus while specificity was 97.7, 98 and $100 \%$ respectively. In the case of S. warneri and S. saprophyticus sensitivity was 50\%, with $100 \%$ specificity in each case. The API Staph 32 demonstrated the highest PVP with the coagulase positive isolates, with S. aureus at $100 \%$ and the SIG group at $83.3 \%$. PVP values could not be interpreted for some coagulase negative species, as the test did not identify the species in some cases. S. xylosus demonstrated a PVP of $27.2 \%$, due to the high number of false positive results and low specificity (83.3\%). Overall, the API Staph 32 had a sensitivity value of $61.5 \%$, a specificity value of $98 \%$ and a PVP value of $80 \%$ compared to identification of staphylococcal species by tuf genotyping.

RapID correctly identified $81.8 \%(9 / 11)$ of the $S$. aureus isolates (sensitivity $81.8 \%$, specificity $100 \%$ ), but misidentified all of the $S$. pseudintermedius isolates. On consideration of ability to identify the SIG group, the RapID test had a sensitivity of $41.6 \%$ and specificity of $95.2 \%$. Only $7 \%$ $(2 / 29)$ of the CONS were correctly identified, $58.6 \%(17 / 29)$ were misidentified, and no result was obtained for $34 \%$ (10/29). When attempting to identify S. xylosus, RapID demonstrated a sensitivity value of $50 \%$, but with a specificity of $66.6 \%$, resulting in a PVP of just $15.8 \%$. Overall, the RapID Staph had a sensitivity value of $32.7 \%$, a specificity value of $95.4 \%$ and a PVP value of $47.2 \%$ compared to identification of staphylococcal species by tuf genotyping.

Staph-Zym correctly identified none of the coagulase positive isolates in this study, and only one of the CONS isolates (Isolate No. 39; S. carnosus). For 11.5\% (6/52) of isolates, Staph-Zym yielded more than one species name. For $44.2 \%$ (23/52) Staph-Zym yielded "no result". 
Table 3 Identification of coagulase positive and negative staphylococcal clinical isolates

\begin{tabular}{|c|c|c|c|c|}
\hline No & tuf & API Staph 32 & RapID & Staph-Zym \\
\hline 1 & S. aureus & S. aureus & S. aureus & no result \\
\hline 2 & S. aureus & S. aureus & S. aureus & S. capitus \\
\hline 3 & S. aureus & S. aureus & S. capitus & no result \\
\hline 4 & S. aureus & S. aureus & S. aureus & no result \\
\hline 5 & S. aureus & S. aureus & S. aureus & no result \\
\hline 6 & S. aureus & S. aureus & S. aureus & no result \\
\hline 7 & S. aureus & S. aureus & S. aureus & S. warneri \\
\hline 8 & S. aureus & S. aureus & S. aureus & S. warneri \\
\hline 9 & S. aureus & S. aureus & S. aureus & S. vitulans \\
\hline 10 & S. aureus st398 & S. aureus & S. aureus & no result \\
\hline 11 & S. aureus st398 & S. aureus & S. gallinarum/xylosus & S. warneri \\
\hline 12 & S. pseudintermedius & S. intermedius & S. xylosus & no result \\
\hline 13 & S. pseudintermedius & S. intermedius & S. gallinarum/xylosus & no result \\
\hline 14 & S. pseudintermedius & S. intermedius & S. xylosus & no result \\
\hline 15 & S. pseudintermedius & S. intermedius & S. intermedius/xylosus & S. capitus \\
\hline 16 & S. pseudintermedius & S. intermedius & S. capitus & no result \\
\hline 17 & S. pseudintermedius & S. intermedius & S. intermedius & S. capitus/hycius \\
\hline 18 & S. pseud/delphini* & S. intermedius & S. gallinarum/xylosus & no result \\
\hline 19 & S. pseudintermedius & S. intermedius & S. epidermidis & S. vitulans \\
\hline 20 & S. pseudintermedius & no result & no result & no result \\
\hline 21 & S. pseudintermedius & S. intermedius & S. intermedius & no result \\
\hline 22 & S. pseudintermedius & S. intermedius & S. intermedius & no result \\
\hline 23 & S. pseud/delphini* & Kocuria rosea & S. hominus/ capitus & S. lentus/fleurettil \\
\hline No & tuf & API 32 & RapID & Staph-Zym \\
\hline 24 & S. felis & S.chromogens & S.chromogenes & S. vitulans \\
\hline 25 & S. felis & S. carnosus & S. intermedius & S. capitus \\
\hline 26 & S. felis & s. xylosus & S. xylosus & no result \\
\hline 27 & S. equorum & S. xylosus & S. xylosus & no result \\
\hline 28 & S. equorum & S. warnerei & S. warnerei & no result \\
\hline 29 & S. equorum & no result & S. xylosus & S.xylosus/scuiri \\
\hline 30 & S. equorum & S. xylosus & S. xylosus & no result \\
\hline 31 & S. equorum & no result & no result & no result \\
\hline 32 & S. equorum & S. xylosus & S. xylosus & S. lentus \\
\hline 33 & S. equorum & S. xylosus & S. xylosus & no result \\
\hline 34 & S. equorum & S. epidermidis & S. epidermidis & no result \\
\hline 35 & S. succinus & S. xylosus & S. xylosus & no result \\
\hline 36 & S.warnerei/ pasteureii & S. warnerei & no result & S. vitulans \\
\hline 37 & S.warnerei/ pasteureii & S. carnosus & no result & S.lentus/fleurettii \\
\hline 38 & S. carnosus / simulans & S. carnosus & no result & S. warnerei \\
\hline 39 & S. carnosus & S. simluans & S. simulans & S. carnosus \\
\hline 40 & S. carnosus & S. carnosus & no result & no result \\
\hline 41 & S. carnosus/simulans & S. simulans & no result & S.capitus/ hyicus \\
\hline 42 & S. carnosus/simulans & S. simulans & no result & S. vitulans \\
\hline 43 & S. carnosus/simulans & s. xylosus & S. xylosus & S. lentus \\
\hline 44 & S. carnosus & no result & no result & no result \\
\hline 45 & S. carnosus/simulans & S. xylosus & S. xylosus & no result \\
\hline 46 & S. carnosus/simulans & S. equorum & S. xylosus & S.xylosus/conhii \\
\hline
\end{tabular}


Table 3 Identification of coagulase positive and negative staphylococcal clinical isolates (Continued)

\begin{tabular}{lllll}
\hline 47 & S. xylosus & S. xylosus & S. xylosus & no result \\
\hline 48 & S. xylosus & S. xylosus & no result & no result \\
\hline 49 & S. xylosus & S. intermedius & no result & S. vitulans \\
\hline 50 & S. xylosus & S. xylosus & S. xylosus & S. scuiri \\
\hline 51 & S. sapraphyticus & S.sapraphytics & S. xylosus & S. lentus \\
\hline 52 & S. sapraphyticus & S. xylosus & S. xylosus & no result \\
\hline
\end{tabular}

${ }^{*}=$ S. pseudintermedius or $\mathrm{S}$. delphini.

\section{Discussion}

The results presented herein emphasise the importance of choosing the correct identification test for accurate speciation of staphylococcal species of animal origin. The accurate identification of staphylococcal species impacts directly and positively on treatment outcomes and on the epidemiological analysis of emerging trends in multi-drug resistant staphylococcal infections in veterinary medicine.

This present study revealed that all three phenotypic test systems yielded inaccurate speciation results when compared to tuf genotyping (Table 3). When considering phenotypic test kits on their own, one must consider the reliability of reading a result with a high "apparent" accuracy. For example, RapID identified one isolate as S. xylosus with a $97 \%$ probability value; however this isolate was subsequently identified by tuf as $S$. pseudintermedius, which could mislead the diagnostician. One of the arguments for using phenotypic test kits is that they are less costly than genotyping. When comparing the costs of phenotyping one must consider the potential consequences of misidentification including unnecessary morbidity and mortality of infected animals.

Of the phenotypic tests utilized, the API Staph 32 correctly identified $100 \%$ of $S$. aureus isolates, $83.3 \%$ of SIG isolates and $31 \%$ of the CONS; the RapID test correctly identified $81.8 \%$ of S. aureus, 33\% of SIG isolates and $6.8 \%$ of the CONS; while the Staph-Zym test correctly identified only $2 \%$ of all isolates. Each of these tests is based on the evaluation of expression of genetically encoded characteristics by bacterial isolates. Inaccurate speciation may be due to variable expression of biochemical traits within species, as previously reported by Blaiotta et al. [8]. This is supported in the present study where it was observed that in each of the test systems, some biochemical tests frequently gave a misleading response for a given species tested. In particular, tests for arginine dihydrolase, arginine arlyamidase, $\beta$-glucuronidase, fructose and mannitol fermentation, novobiocin resistance and nitrate reduction, were observed in one or more systems to generate a response contrary to the expected result for a given species (data not shown). In addition, species identification kits such as these are manufactured for the human diagnostics market and are interpreted against databases with reference strains of human origin. This suggests that the reproducibility and therefore reliability of these tests is questionable when applied to veterinary isolates. When the identification of $S$. aureus by the three phenotyping test kits is considered, it was observed that while all of the $S$. aureus isolates were identified by API Staph 32, they were not consistently identified by either Rap-ID or Staph-Zym, demonstrating a lack of correlation between tests systems for a

Table 4 Sensitivity, specificity and predictive value positive of API Staph 32, RapID Staph and Staph-Zym tests showing individual results for species isolated in this study and overall values for test kits

\begin{tabular}{|c|c|c|c|c|c|c|c|c|c|}
\hline Species & & Staph & & & RapID & & & ph-Zy & \\
\hline & Sens $^{1}$ & Spec $^{2}$ & $P V P^{3}$ & Sens & Spec & PVP & Sens & Spec & $\overline{P V P}$ \\
\hline Overall & 61.5 & 98 & 80 & 32.7 & 95.4 & 47.2 & 1.9 & 99 & 20 \\
\hline S. aureus & 100 & 100 & 100 & 81.8 & 100 & 100 & 0 & 100 & $n / a$ \\
\hline$\overline{S I G}$ & 83.3 & 95 & 83.3 & 41.6 & 95.2 & 71.4 & 0 & 95 & $\mathrm{n} / \mathrm{a}$ \\
\hline S. felis & 0 & 98 & $n / a^{4}$ & 0 & 98 & $\mathrm{n} / \mathrm{a}$ & 0 & 98 & $\mathrm{n} / \mathrm{a}$ \\
\hline S. equorum & 0 & 97.7 & $\mathrm{n} / \mathrm{a}$ & 0 & 100 & $\mathrm{n} / \mathrm{a}$ & 0 & 100 & $\mathrm{n} / \mathrm{a}$ \\
\hline S. succinus & 0 & 100 & $\mathrm{n} / \mathrm{a}$ & 0 & 100 & $\mathrm{n} / \mathrm{a}$ & 0 & 100 & $\overline{n / a}$ \\
\hline S. warneri & 50 & 98 & 50 & 0 & 98 & $\mathrm{n} / \mathrm{a}$ & 0 & 98 & $\mathrm{n} / \mathrm{a}$ \\
\hline S. carnosus & 66.6 & 95.3 & 75 & 11.1 & 100 & 100 & 11.1 & 100 & 100 \\
\hline S. xylosus & 77 & 83.3 & 27.2 & 50 & 66.6 & 15.8 & 0 & 66.6 & $\mathrm{n} / \mathrm{a}$ \\
\hline S. saprophyticus & 50 & 100 & 50 & 0 & 100 & $\mathrm{n} / \mathrm{a}$ & 0 & 100 & $n / a$ \\
\hline
\end{tabular}

Values calculated using tuf genotyping as a reference method.

${ }^{1}$ Sensitivity ${ }^{2}$ specificity ${ }^{3}$ predictive value positive ${ }^{4}$ not applicable. 
commonly isolated species. In addition, of the eight S. equorum isolates identified by genotyping, API Staph 32 identified four as $S$. xylosus, one as S. epidermidis, one as $S$. warneri and did not identify two, suggesting within species variability for the test system. With respect to the newly recognised Staphylococcus intermedius group [30], the failure of the phenotypic test kits to correctly speciate members of this group is of concern due to the relevance of S. pseudintermedius, not only as a veterinary pathogen, but as a source of nosocomial infection [31].

DNA sequencing of housekeeping genes is regularly used to definitively type staphylococcal isolates, [9,18-21,32]. In this study, tuf and $r p o B$ gene segments were amplified by PCR and sequenced according to published methods $([9,19]$, respectively). Both genes constitute more discriminatory targets than the 16S-rRNA gene to differentiate closely related staphylococcal species. The results of this study demonstrated $100 \%$ accuracy for reference strains using tuf genotyping. Among the clinical isolates, 23 were identified by tuf genotyping as coagulase positive species (Table 3). Interestingly, five of these isolates failed to coagulate in both the tube and slide agglutination tests. These findings are not atypical however. According to Murray et al. [6] up to $30 \%$ of S. aureus field isolates fail to display coagulase activity. Reduced coagulase activity in $S$. aureus is also reported to be associated with reduced susceptibility to vancomycin [33]. It is worth noting that in the present study, each of the four S. pseudintermedius isolates which failed to coagulate also showed reduced susceptibility to vancomycin (data not shown), tuf genotyping identified 29 coagulase negative isolates and 5 distinct species (Table 3). The species $S$. carnosus and S. simulans, however, could not be definitively differentiated from each other by tuf genotyping. Likewise, S. warneri and S. pasteuri were not differentiated by this method. Previous authors have documented a close phylogenetic link between these pairs of species $[29,34,35]$ and the current findings support this.

All three of the reference SIG species were identified correctly by tuf genotyping (S. intermedius, S. delphini, S. pseudintermedius). Ten of the clinical isolates were identified as S. pseudintermedius, but for two additional isolates, tuf could not differentiate between $S$. pseudintermedius and S. delphini. In an attempt to clarify the identity of these two isolates, both of canine origin, rpoB sequencing and PCR-RFLP of the pta gene were performed. Both isolates were confirmed as S. pseudintermedius by the two methods. Given the clinical significance of S. pseudintermedius in veterinary medicine, and the published evidence that MRSP (methicillin resistant S. pseudintermedius) is emerging as a nosocomial infection, the importance of an accurate identification is paramount. Our findings suggest the use of $r p o \mathrm{~B}$ genotyping or PCR-RFLP of the pta gene as a confirmatory method for discriminating between SIG isolates until a larger cohort of these species are entered into the tuf gene database, thereby enhancing its accuracy.

\section{Conclusion}

Of the three biochemical tests used, the API Staph 32 test performed with the highest degree of accuracy for the coagulase positive Staphylococci. When compared to tuf genotyping all three of the rapid biochemical tests performed poorly for the speciation of coagulase negative Staphylococci. This study highlights the importance of choosing the correct identification test for accurate speciation of staphylococcal species of companion animal origin, as failure to correctly identify specific pathogens may impact on subsequent antimicrobial interventions.

\section{Competing interests}

The authors declare no competing interests.

\section{Authors' contributions}

LG collected all experimental the data, participated in the study design, sequence alignment and drafting of the manuscript. MB participated in the study design, sequence alignment and drafting of the manuscript. AF participated in the design of the study and drafting the manuscript. NR participated in the study design and drafting of the manuscript. All authors read and approved the final manuscript.

\section{Acknowledgements}

The authors would like to acknowledge the help of Dr. Arshnee Moodley, University of Copenhagen, in the preparation of this paper, and for the donation of some of the reference strains used. We would also like to thank Dr. Damien Brady, Athlone Institute of Technology, and Ms. Yvonne Abbott, University College Dublin, for the donation of reference strains. We would like to thank Dr. Rory Breathnach, University College Dublin, for his contribution to this research work. This work was jointly funded by Irish Strand 1 Technological Sector Research Funding programme and by the President's Seed Funding Initiative at Athlone Institute of Technology, Ireland.

Received: 12 January 2013 Accepted: 2 April 2013

Published: 1 May 2013

\section{References}

1. Guardabassi L, Loeber ME, Jacobson A: Transmission of multiple antimicrobial-resistant Staphylococcus intermedius between dogs affected by deep pyoderma and their owners. Vet Microbio/ 2004, 98:23-27.

2. Leonard FC, Abbott Y, Rossney A, Quinn PJ, O'Mahony R, Markey BK: Methicillin-resistant Staphylococcus aureus isolated from a veterinary surgeon and five dogs in one practice. Vet Res 2006, 58:155-159.

3. Malik S, Peng H, Barton MD: Partial nucleotide sequencing of the mecA genes of Staphylococcus aureus isolates from cats and dogs. J Clin Microbiol 2006, 44:413-416.

4. Weese JS, Dick H, Willey BM, McGeer A, Kreiswirth BN, Innis B, Low DE: Suspected transmission of methicillin-resistant Staphylococcus aureus between domestic pets and humans in veterinary clinics and in the household. Vet Microbiol 2006, 115:148-155.

5. Sampimon AC, Lam TG, Mevius DJ, Schukken YH, Zadoks RN: Antimicrobial susceptibility of coagulase negative staphylococci isolated from bovine milk samples. Vet Microbiol 2011, 150:173-179.

6. Murray PR, Baron EJ, Jorgensen JH, Landry ML, Pfaller MA: Manual of Clinical Microbiology, 9th Edition. American Society Microbiology 2007:390-411.

7. Coppola R, Mauriello G, Aponte M, Moscheitti G, Villani F: Microbial succession during ripening of Naples-type salami, a southern Italian fermented product. Meat Sci 2000, 56:321-329. 
8. Blaiotta G, Pennacchia C, Villani F, Ricciardi R, Tofalo R, Parente E: Diversity and dynamics of communities of coagulase-negative staphylococci in traditional fermented sausages. J Appl Microbiol 2004, 97:271-284.

9. Heikens E, Fleer A, Paauw A, Florijn A, Fluit AC: Comparison of genotypic and phenotypic methods for species-level identification of clinical isolates of coagulase-negative staphylococci. J Clin Microbiol 2005, 43:2286-2290.

10. Capurro A, Artursson K, Waller KP, Bengtsson B, Ericsson-Unnerstad H, Aspán A: Comparison of a commercialized phenotyping system, antimicrobial susceptibility testing, and tuf gene sequence-based genotyping for specieslevel identification of coagulase-negative staphylococci isolated from cases of bovine mastitis. Vet Microbiol 2009, 134:327-233.

11. Zadoks RN, Watts JL: Species identification of coagulase-negative staphylococci: genotyping is superior to phenotyping. Vet Microbiol 2009, 134:20-28.

12. Blaiotta G, Pennachia C, Ercolin D, Moschetti G, Villani F: Combining denaturing gradient gel electrophoresis of 16S rDNA V3 region and 16S$23 \mathrm{~S}$ rDNA spacer region polymorphism analyses for the identification of staphylococci from Italian fermented sausages. Sys Appl Microbiol 2003, 26:423-433.

13. Burriel AR, Scott M: A comparison of methods used in species identification of coagulase-negative staphylococci isolated from the milk of sheep. Vet J 1998, 155:183-188.

14. Jousson O, Di Bello D, Vanni M, Cardini G, Soldani G, Pretti C, Intorre L: Genotypic versus phenotypic identification of staphylococcal species of canine origin with special reference to Staphylococcus schleiferi subsp. coagulans. Vet Microbiol 2007, 123:238-244.

15. Thorberg BM, Brändström B: Evaluation of two commercial systems and a new identification scheme based on solid substrates for identifying coagulase-negative staphylococci from bovine mastitis. J Vet Med 2000, 47:683-691.

16. Taponen S, Pyörälä S: Coagulase-negative staphylococci as cause of bovine mastitis- not so different from Staphylococcus aureus? Vet Microbiol 2009, 134:29-36.

17. Park JY, Fox LK, Seo KS, McGuire MA, Park YH, Rurangirwa FW, Sischo WM, Bohach GA: Comparison of genotypic and phenotypic methods for the phenotypic identification of coagulase-negative Staphylococcal isolates from bovine intramammary infections. Vet Microbiol 2010, 147:142-148.

18. Shah MM, lihara H, Noda M, Song SX, Nhung PH, Ohkusu K, Kawamura Y, Ezaki T: DnaJ gene sequence-based assay for species identification and phylogenetic grouping in the genus Staphylococcus. Int J Syst Evol Microbiol 2007, 57:25-30

19. Drancourt M, Raoult D: RpoB Gene Sequence-Based Identification of Staphylococcus Species. J Clin Microbiol 2002, 40:1333-1338.

20. Becker K, von Eiff C, Keller B, Brück M, Etienne J, Peters G: Thermonuclease gene as a target for specific identification of Staphylococcus intermedius isolates: use of a PCR-DNA enzyme immunoassay. Diagn Microbiol Infect Dis 2005, 51:237-244.

21. Poyart C, Quesne G, Boumaila C, Trieu-Cuot P: Rapid and accurate specieslevel identification of coagulase-negative staphylococci by using the sodA gene as a target. J Clin Microbiol 2001, 39:4296-4301.

22. Bergeron M, Dauwalderm O, Gouym M, Freydiere AM, Bes M, Meugnier H, Benito $Y$, Etienne J, Lina G, Vandenesch F, Boisset S: Species identification of staphylococci by amplification and sequencing of the tuf gene compared to the gap gene and by matrix-assisted laser desorption ionization time-of-flight mass spectrometry. Eur J Clin Microbiol Infect Dis 2010, 30:343-354.

23. Hwang SM, Kim MS, Park KU, Song J, Kim EC: tuf gene sequence analysis has greater discriminatory power than 16S rRNA sequence analysis in identification of clinical isolates of coagulase-negative staphylococci. J Clin Microbiol 2011, 49:4142-4149.

24. Blaiotta G, Fusco V, Ercolini D, Pepe O, Coppola S: Diversity of Staphylcoccus species strains based on partial kat (catalase) gene sequences and design of a PCR-restriction fragment length polymorphism assay for identification and differentiation of coagulase-positive species (S. aureus, S. delphini, S. hyicus, S. schleiferi subsep. coagulans). J Clin Microbio/ 2010, 48:192-201.

25. Sasaki T, Tsubakishita S, Tanaka Y, Ohtsuka M, Hirotaki S, Kawakami T, Fukata T, Hiramatsu K: Multiplex- PCR method for species identification of coagulase positive staphylococci. J Clin Microbiol 2010, 48:765-769.

26. NCBI; 2012. http://www.ncbi.n/m.nih.gov/Taxonomy/Browser/wwwtax.cgi? $i d=1279$.
27. Bannoehr J, Franco A, lurescia M, Battisti A, Fitzgerald JR: Molecular diagnostic identification of Staphylococcus pseudintermedius. J Clin Microbiol 2009, 47:469-471.

28. Kadlec K, Rohde J, Schwar S: Identification and presence of different staphylococcal species within the Staphylococcus intermedius group among various animal hosts. In Methicillin-resistant Staphylococci in animals: Veterinary and public health implications. ASM conference; 2009:42.

29. Ghebremedhin B, Layer F, König W, König B: Genetic classification and distinguishing of Staphylococcus species based on different partial gap, 16S rRNA, hsp60, rpoB, sodA, and tuf gene sequences. J Clin Microbiol 2008, 46:1019-1025.

30. Devriese LA, Vancanneyt $M$, Baele M, Vaneechoutte $M$, De Graef $E_{i}$ Snauwaert C, Cleenwerck I, Dawyndt P, Swings J, Decostere A, Haesebrouck F: Staphylococcus pseudintermedius sp. nov., a coagulase-positive species from animals. Int J Syst Evol Microbiol 2005, 55:1569-1573.

31. Paul NC, Moodley A, Ghibaudo G, Guardabassi L: Carriage of methicillinresistant Staphylococcus pseudintermedius in small animal veterinarians: indirect evidence of zoonotic transmission. Zoonoses Public Health 2011, 58:533-539.

32. Barros EM, lório NL, Bastos Mdo C, dos Santos KR, Giambiagi-deMarval M: Species-level identification of clinical staphylococcal isolates based on polymerase chain reaction-restriction fragment length polymorphism analysis of a partial groEL gene sequence. Diagn Microbiol Infect Dis 2007, 59:251-257.

33. Moreira B, Boyle-Varva S, deLonge BL, Daum RS: Increased production of penicillin binding protein 2 , increased detection of other penicillinbinding proteins, and decreased activity associated with glycopeptide resistance in Staphylococcus aureus. Antimicrob Agents Chemother 1997, 41:1788-1793.

34. Mellman A, Becker $K$, van Eiff C, Keckevoet U, Schumann P, Harmsen D: Sequencing and Staphylococci identification. Emerg Infect Dis 2006, 12:333-336.

35. Shin JH, Kim SH, Jeong HS, Oh SH, Kim HR, Lee JN, Yoon YC, Kim YW, Kim $\mathrm{YH}$ : ( Identification of coagulase-negative staphylococci isolated from continuous ambulatory peritoneal dialysis fluid using $16 \mathrm{~S}$ ribosomal RNA, tuf, and sodA gene sequencing. Perit Dial Int 2011, 31:340-346.

doi:10.1186/2046-0481-66-7

Cite this article as: Geraghty et al:: Investigations on the efficacy of routinely used phenotypic methods compared to genotypic approaches for the identification of staphylococcal species isolated from companion animals in Irish veterinary hospitals. Irish Veterinary Journal 2013 66:7.

\section{Submit your next manuscript to BioMed Central and take full advantage of:}

- Convenient online submission

- Thorough peer review

- No space constraints or color figure charges

- Immediate publication on acceptance

- Inclusion in PubMed, CAS, Scopus and Google Scholar

- Research which is freely available for redistribution 